\title{
LOS MODELOS DIDÁCTICOS EN LA FORMACIÓN DE DOCENTES UNIVERSITARIOS, LA EMERGENCIA DE LOS SABERES-OTROS (TECNOLÓGICO Y AMBIENTAL) EN PANDEMIA DE LA COVID-19
}

\section{THE DIDACTIC MODELS IN THE TRAINING OF UNIVERSITY TEACHERS, THE EMERGENCY OF THE KNOWLEDGE-OTHERS (TECHNOLOGICAL AND ENVIRONMENTAL) IN THE COVID-19 PANDEMIC}

\section{Claudia María Cardona ${ }^{1}$}

Edwin Rivas Trujillo ${ }^{2}$

Universidad Distrital Francisco José de Caldas

\section{RESUMEN}

La comunicación tiene como propósito presentar esos saberes-otros que emergen como importantes en Pandemia de la Covid-19 y que hacen parte de la formación del Docente Universitario. El inicio es el modelo pedagógico/

$1 \quad$ Docente Investigador. Facultad del Medio Ambiente y Recursos Naturales. Universidad Distrital Francisco José de Caldas. Ingeniera Agrícola, MsC. en Recurso Hídricos. (c) PhD en Educación. https://orcid.org/0000-00015430-1392 cmcardonal@udistrital.edu.co

2 Docente Investigador. Facultad de Ingeniería. Universidad Distrital Francisco José de Caldas. Ingeniero Electricista - MsC y PhD. En Ingeniería Eléctrica, Electrónica y Automática. https://orcid.org/0000-0003-2372-8056 erivas@udistrital.edu.co

3 Docente Investigador. Facultad de Ingeniería. Universidad Distrital Francisco José de Caldas. Ingeniería Industrial, MsC en ingeniería Industrial. PhD en Educación.https://orcid.org/0000-0002-1170-4332 mariaramirezsanchez07@ gmail.com

didáctico de (Shulman, 1987 -PCK), y la fusión de muchos que mezclan de manera independiente esos saberes-otros, como el de (Mishra \& Koehler, 2006 -TPACK y el de Zhou, 2015EPACK). A manera de escenario apuesta para la educación del siglo XXI situada en un horizonte de nuevas realidades globales, nos ubica ante retos inéditos, por ejemplo, 'el tecnológico' y ‘lo ambiental', esos llamados los saberes-otros, que, desde la mirada de una inclusión de una educación superior, compromete a un cambio de paradigma que favorece la interdisciplinariedad y el aprendizaje investido.

\section{PALABRAS CLAVE:}

Modelos Didácticos: CDC, TPACK, EPACK; Formación Docente; Pandemia de la Covid-19. 


\section{ABSTRACT}

The purpose of the communication is to present those knowledge-others that emerge as important in the Covid-19 Pandemic and that are part of the University Teacher training. The beginning is the pedagogical / didactic model of (Shulman, 1987 -PCK), and the fusion of many that independently mix those knowledge-others, such as that of (Mishra \& Koehler, 2006 -TPACK and that of Zhou, 2015- EPACK). As a scenario bet for the education of the XXI century located in a horizon of new global realities, it places us before unprecedented challenges, for example, 'the technological' and 'the environmental', those called the knowledge-others, which, from the Looking at an inclusion of a higher education, commits to a paradigm shift that favors interdisciplinary and invested learning.

\section{KEY WORD:}

Teaching Models: CDC, TPACK, EPACK;

\section{INTRODUCCIÓN}

En respuesta a la Pandemia de la Covis-19, la Educación Superior, debió enfrentar el reto de comenzar a explorar los saberes-otros (más allá de los disciplinares) para un mejoramiento del quehacer docente. Es desde ahí, que surgen los marcos de entendimiento en los cuales se habla de la función docente que, más que ser un conjunto de saberes paralelos o independientes, busca integrar con eficacia el saber disciplinar con el proceso de enseñanza-aprendizaje, de manera que, articulados, permita generar un conocimiento integral que contribuya a los resultados de aprendizaje en los estudiantes.

El artículo tiene un enfoque reflexivo-crítico, es histórico-hermenéutico y permite abordar el estudio de caso como metodología para analizar e interpretar 'las prácticas de enseñanza y saber pedagógico' de los docentes en clave

de las perspectivas de la Educación Superior. Se asume la práctica (el saber didáctico) no como algo extraordinario, sino como parte del ejercicio cotidiano del 'ser-hacer-pensar y sentir' del docente universitario. Con los saberesotros (tecnológico y el ambiental) es necesario interpelar las acciones instrumentales $y$ requeridas para el aprendizaje invertido y de las dimensiones éticas, políticas y culturales, para el segundo caso. Se justifica la comprensión de este tipo de educación, porque es incluyente y configura entornos de aprendizaje y de formación del docente, por un lado, y a su vez, un contexto de sustentabilidad, por el otro lado.

El objetivo central del artículo de reflexión crítica es la apropiación de esos saberes-otros que se han incluido en los modelos didácticos en la perspectiva universitaria para la formación integral de los docentes. Es en el uso de estrategias, recursos y métodos, es decir, la concreción de las prácticas de enseñanza que se privilegia entre otros, la pregunta, la creación de situaciones problema, los estudios de caso como método, la configuración de expertos, y el uso de todas las tecnologías disponibles en la web (Londoño, 2014: 32).

La importancia del artículo radica en los resultados del proceso bibliográfico que permite reconocer que la práctica del Docente Universitario requiere de múltiples saberes además del disciplinar, que abarca diversas dimensiones que implica el dominio de competencias y habilidades. Es desde este punto de partida que se tiene como desafío, la integración los modelos didácticos que hacen parte del proceso de formación permanente de los Docentes Universitarios.

Se requiere saber de la disciplina (conocimiento del contenido (KK), saber de lo pedagógico/ didáctico (conocimiento pedagógico $\mathrm{PC}, \quad \mathrm{O}$ conocimiento didáctico DC), no como una teoría descontextualizada, sino en el ámbito de la 
Educación Superior y de los saberes-otros: la realidad socio-cultural, económica-política, eco sistémica-ética/estética, que permea el mundo universitario y todos los actores involucrados y por último el saber tecnológico, que es el motor del mundo simulado y de cierre de brechas.

Para adquirir el dominio de competencia y elaborar argumentos científicos, tecnológicos y ambientales, se hace necesario un mínimo de conciencia y sensibilización, comprensión y empatía, participación y decisión entre otros. Se requiere de un 'hacer' que asume lo didáctico más allá de la aplicación estrategias y parta de los problemas reales del mundo de la vida.

El conocimiento del mundo de la vida y sus limitaciones incluye una serie de bases conceptuales que definen lo que significa aprender, puesto que los docentes deben comprender la naturaleza la didáctica a utilizar, de los diferentes tipos de evaluación y un conjunto de estrategias pedagógicas para que los estudiantes alcancen los resultados de aprendizaje.

Para hablar de los 'saberes-otros', se cita a Morín, (1999), el evento de la Covid-19 de principios del año 2019 y que no termina, cambio de manera radical la forma de ver el mundo y la preocupación por una educación de calidad. Se comenzó con una nueva normalidad, partiendo de un paradigma crítico-interpretativo, que exige asumir unas bases teóricas interdisciplinares. El caso de la Pandemia de la Covid-19, será objeto de muchas investigaciones desde diferentes miradas, una de ella la educativa (Ramírez, Rivas y Cardona. 2019). La Docencia Universitaria implica comprender la complejidad de la práctica educativa que está vinculada con una serie de instrumentos metodológicos y reflexiones sobre el quehacer de la enseñanza. Las actitudes de los docentes universitarios durante la Covid-19, se convirtieron en oportunidades para transformar los procesos de enseñanza-aprendizaje. (Cardona, Ramírez \& Rivas, 2020).

Los procesos de enseñanza-aprendizaje reconoce el papel de la didáctica en la inclusión de estos saberes-otros (tecnológico-ambiental), que vincula no solo el saber disciplinar, sino que está vinculado también a teorías educativas, modelos pedagógicos/didácticos, paradigmas de aprendizaje entre otros, que se relacionan con los proyectos curriculares y la concepción de la Educación Superior.

Se presenta como marco referencial los siete saberes de la educación del futuro una propuesta de (Morín, 1999) para estructurar un marco conceptual sobre los cuatro (4) modelos didácticos básicos de formación del Docente Universitario que incluye la disciplinar, pedagógico/didáctico y los saberes-otros: Tecnológico y el Ambiental.

\section{METODOLOGÍA DEL ARTíCULO}

La metodología para el desarrollo del artículo es el Estudio de Caso único (Yin, 2014). Para Ramírez, Rivas y Cardona. (2019) es una herramienta útil en la investigación, y su validez radica en que a través del mismo se mide y registrala conducta de los docentes universitarios incluidas en el fenómeno estudiado, que es el proceso de enseñanza-aprendizaje. Se plantea el estudio de caso único que centrados en el 'estudio de descripciones', en este caso se propone como objetivo específico, a partir de una consigna que permita el: análisis, identificación y descripción de los puntos clave constitutivos de una situación dada y tengan la posibilidad de debatir y reflexionar de manera crítica, y desde las distintas perspectivas. Finalmente, pretenden la reflexión y el estudio sobre los principales temas teórico-prácticos que se derivan de la situación estudiada. No se pretende, pues, llegar al estudio y al planteamiento de soluciones, se centran en 
aspectos exclusivamente descriptivos. Este tipo de casos, que tiene entidad propia en cuanto análisis descriptivo, constituye el punto de partida de los subtipos de estudios de caso múltiples.

El estudio de caso tiene la siguiente consigna: ¿los modelos didácticos en la formación de docentes universitarios, y esos saberes-otros (tecnológico y ambiental) en pandemia de la covid-19, han posibilitado el ser, saber y hacer en la Educación Superior y son suficientes para la nueva normalidad?

El diseño de estudio de casos como estrategia metodológica sirve para estudiar la didáctica de la docencia universitaria antes, durante y cómo horizonte en la Covid-19. Los resultados son eficientes para incluir en la reforma universitaria la necesidad de permanente formación de los Docentes Universitarios y proponer recomendaciones y cambios en las mismas, evaluar programas, construir instrumentos de investigación y sirve para fines políticos y científicos.

Los hallazgos de la investigación en este tipo de diseño, establecen una formulación teórica sobre la realidad en contexto. El estudio de casos es una metodología apropiada para aprehender la realidad de una situación estratégica en la Educación superior, en los que se requiera explicar, por ejemplo, la necesidad de hablar de Didáctica Universitaria, dado que de manera directa está afectada por las teorías del aprendizaje. (Ramírez, Rivas y Cardona; 2019)

Para describir el estudio de caso asociado a este artículo de reflexión crítica sobre la consigna dada se presentan los tres (3) pilares del caso:

1. La teoría fundamentada en los 7 saberes de la Educación del Futuro propuestos por (Morín, 1999), 20 años después.
2. Los cuatro (4) modelos didácticos que sirven como referente de la formación en la didáctica universitaria, cuyo objetivo es el análisis de lo que sucede en la práctica de la enseñanza, da cuenta de una reflexión sistemática sobre la acción.

3. Los Escenarios apuesta, que vincula la estrategia didáctica para la nueva normalidad post-Covid-19, que serán presentadas a manera de reflexión final.

\section{MARCO DE REFERENCIA}

Las distintas miradas para la formación de los Docentes Universitarios se han dado según los autores de referencia, aquí se presenta el enfoque de la didáctica. Se asume como una acción instrumental que da sentido a las prácticas de los docentes asumiendo la educabilidad del ser humano, que atiende procesos de desarrollo personal y cultural y sus posibilidades de formación y aprendizaje. $Y$ la enseñabilidad de las disciplinas y saberes-otros producidos por la humanidad en un marco de sus dimensiones tecno-científicas, socio-sistémicas, ecosistémicas y las de más alto nivel, las culturales, éticas y políticas. La interdisciplinariedad y los modelos que presentan una estructura del deber-ser, son base para la reflexión crítica que comienza con la descripción de los siete (7) saberes de la educación del futuro de Morín (1999) y se desarrollan con los modelos de Shulman (1986), Koehler \& Mishra $(2009,2015)$ y su modificación y el de Zhou (2018), para incluir en el de Shulman los saberes-otros y comenzar a reflexionar sobre la consigna inicial.

Parte I. La Educación del futuro según Morín (1999) se fundamenta en: 1) cubrir la ceguera del conocimiento, 2) trabajar por un conocimiento pertinente a la realidad en contexto, 3) enseñar sobre la condición humana, 4) abordar el enfoque de identidad, 5) haciendo frente a las incertidumbres, 6) enseñar para comprender las relaciones y las escalas desde donde emergen 
los problemas del mundo y, por último, 7) la inclusión de la ética. El abordaje del estudio de caso implica una descripción densa y corta de cada elemento para su reflexión crítica.

\section{LA EDUCACIÓN QUE CURE LA CEGUERA DEL CONOCIMIENTO.}

La Educación superior debe contar con la posibilidad de estudiar los errores, o niveles de significancia de las acciones de cada disciplina frente a la realizar de la transformación del entorno natural. En este actuar sin medida, agotar el planeta y generar perturbación en el entorno natural, a tal estado que ya no se habla de crisis ambiental, sino de riesgo a desaparecer; nos conduce a una fragilidad de juicios sobre lo que se enseña y debe ser aplicado para una armonía con la propia cultura y la influencia de los proyectos de desarrollo tecnológico, que exigen una afectividad ambiental.

La primera competencia del Docente

Universitario será enseñar un 'conocimiento capaz de criticar el propio conocimiento'. No existen verdades absolutas y la flexibilidad en pensar críticamente, conduce a la corrección de errores. El escenario apuesta de la educación del futuro según Morín, (1999) es fortalecer en los estudiantes la capacidad para "detectar y subsanar los errores e ilusiones del conocimiento y, al mismo tiempo, enseñarles a convivir con sus ideas, sin ser destruidos por ellas".

\section{LA EDUCACIÓN CON UN CONOCIMIENTO PERTINENTE.}

Ante un inmenso mar de informaciones, que no es conocimiento, se requiere la comprensión de los problemas del mundo para trabajar en cada tiempo en sus soluciones, se requiere que cada espacio educativo superior se un campo del conocimiento interdisciplinar de múltiples relaciones complejas entre cada una de las actividades, componentes, subsistemas del sistema en su total y completa dimensión. La base es una reflexión crítica que configure la capacidad de formular los problemas del mundo por la existencia humana y poderlos resolver. Se deben trabajar la complejidad desde lo amplio y profundo de lo pertinente y simple.

\section{LA EDUCACIÓN QUE ENSEÑA LA CONDICIÓN HUMANA}

La Educación Superior del siglo XXI, debe hace énfasis en la otredad y alteridad, como base del respeto por la diferencia, por un diálogo de saberes que permite situar al ser humano en el único planeta que conocemos. Al enseñar sobre la condición humana, se hace necesario que sea entendido el entorno no solo como el proveedor de las necesidades y satisfactores humanos, sino cómo el medio que sustenta la vida de todos, las preguntas que orientar el ser-estar-hacer-habitar, permite comprender el conjunto de significados sobre las formas de habitar la tierra, para conservar la unidad de los seres humanos y propender por el Buen vivir/Vivir Bien. Este conjunto de conceptos (necesidades, satisfactores, sustentabilidad, buen vivir) forman parte de una mirada ética y cultural que debe ser enseñada desde esos saberes-otros, "lo ambiental" como ciudadanos de la tierra.

\section{LA EDUCACIÓN DESDE EL ENFOQUE DE LA IDENTIDAD}

Las diásporas en el mundo produjeron una revolución tecnológica que logró mezclar culturas y unir lo disperso. Ahora bien, la pérdida de identidad sobre una tierra local, bajo la premisa de ser planetarios, introdujo el desarrollo y crecimiento económico, que cómo consecuencia la fragilidad de lo afectivo, moral y valores por las tradiciones culturales quede relegado a un segundo plano. La Educación superior tiene el compromiso de recuperar el desarrollo a escala humana para sustentar la vida en el planeta, que solo es posible lograrlo 
con un verdadero y profundo sentido de pertenencia, que co-construya una conciencia medio ambiental con participación de todos.

\section{LA EDUCACIÓN HACIENDO FRENTE A LAS INCERTIDUMBRES}

Los Modelos son un constructo del ser humano, la naturaleza es un fractal, por tal razón, hablar de incertidumbre es un proceso netamente estadístico-determinístico-estocástico, para reducir lo impredecible del futuro por venir. Aunque es evidente que para Morin, (1999), la incertidumbre aquí es sobre los criterios de validez del conocimiento que se optimiza de acuerda a las alternaciones de decisiones planteadas en función de un tema, en este caso el sistema de la tierra y sus habitantes que lo transforma.

La Educación Superior presenta un horizonte de incertidumbres sobre las realidades en contexto y es en clave del conocimiento disciplinar y esos saberes-otros que pueden ser anticipados. Para el caso del contexto de la Educación Superior forman parte del campo de la didáctica y el contexto.

\section{LA EDUCACIÓN ESTÁ PARA ENSEÑAR A COMPRENDER: RELACIONES Y ESCALAS}

La Educación posmoderna, está acompañada de múltiples relaciones sociales que deben estudiadas desde lo interpersonal e intergrupal a diferentes escalas planetarias en contexto, local, regional, nacional y global. Lo importante son los protocolos que permitan replicar y escalar las diferentes cosmovisiones desde lo socio-cultural, económico-político y ecológicoético. La Educación Superior debe propender por el fortalecimiento de la capacidad de empatía y compasión, de tal manera que exista tolerancia el que piensa diferente y valoremos la dignidad humana.

\section{LA EDUCACIÓN DEBE ENSEÑAR SOBRE LA ÉTICA}

La relación sociedad-naturaleza es fundamental para entender la racionalidad de una sociedad que se piensa el centro de todo y una dependencia total y absoluta a los elementos de la naturaleza. Es claro que para hablar de ética es importante incluir la estética y la diversidad de posibilidades políticas (intenciones sociales) para incluir los saberes-otros en los nuevos discursos democráticos. La Educación superior deberá permear el compromiso por el cuidado el planeta, como parte de la vida misma.

Parte II. Los Modelos didácticos que hacen parte de esta experiencia reflexiva son el puente entre la enseñabilidad y la educabilidad en un proceso formativo del Docente Universitario. La didáctica exige la superación de su reducción a la instrumentación y al uso de métodos eficaces y adecuados, va más allá y vincula el conocimiento en relaciones y posibilidades que transforman las acciones de la práctica. En la Pandemia de la Covid-19, la reinvención de los docentes con la inclusión de esos saberesotro fue de gran impacto y los procesos de adaptación y cambio podrán ser evaluados con el aprendizaje de los estudiantes. A continuación se describen de manera sucinta los cuatro (4) modelos ya citados por (Cardona, et al, 20219) para reflexión sobre la implicaciones en el contexto de la Educación Superior.

\section{EL MODELO CONOCIMIENTO DIDÁCTICO DEL CONTENIDO- CDC}

El Conocimiento Pedagógico del Contenido PCK, presentado por Shulman (1986), Figura 1. Modelo de Shulman. conocido también como Conocimiento Didáctico del Contenido - CDC (Mora y Parga, 2007). Se establece como uno de los tres tipos de conocimiento existentes en el aula, sumado a el conocimiento cotidiano del estudiantado y el conocimiento científico elaborado en cada disciplina. Desde sus 
inicios hasta el día de hoy, se ha establecido como un modelo teórico para entender la enseñanza de los contenidos, diversos casos se han documentado al respecto en diferentes áreas del conocimiento de las disciplinas; así mismo para el caso puntual de la química son destacados los estudios de Garritz y TrinidadVelazco (2004).

Figura 1. Pedagogical Content Knowledge (PCK).

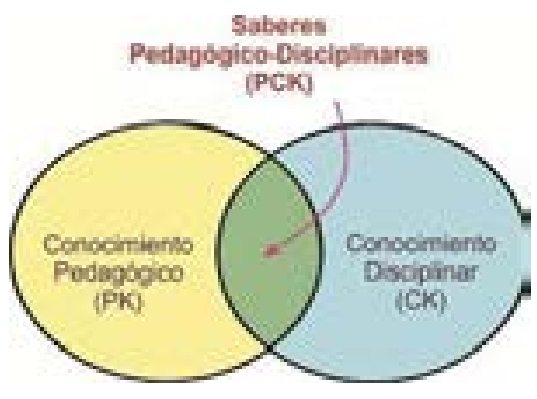

Fuente: Shulman (1986)

El modelo CDC surge como producto de una serie de cambios y evoluciones de diversas propuestas, en el cual se evidencia el importante rol del docente en cuanto domina su disciplina $y$, posee la capacidad de trabajar de manera mancomunada en la formulación de currículos y prácticas que transforman el conocimiento científico en conocimiento enseñable en las aulas. En épocas de Pandemia de la Covid-19, los modelos didácticos debieron migrar a nuevas dimensiones e incorporar esos seres-otros (el tecnológico y el ambiental, por ejemplo).

Para Shulman, (1986) el Programa de investigación sobre el Conocimiento Pedagógico del Contenido, fue el énfasis en la importancia de las investigaciones sobre las formas de pensamiento, creencias y teorías que hacen parte del pensamiento del docente, determinando su concepción acerca de los conocimientos, las ciencias, los procesos de enseñanza-aprendizaje, y la relación teóricapráctica de sus acciones profesionales como docente. Esfuerzo que continuo en España dando origen a el Conocimiento Didáctico del Contenido - CDC y que se ha basado en promover el campo de la didáctica, la enseñabilidad del conocimiento, en disciplinas específicas, todo lo cual busca la optimización del quehacer docente mediante el diseño de planes de desarrollo profesional del mismo, que deben ir de la mano con el diseño curricular.

\section{EL MODELO TPACK EN LA APROPIACIÓN DE CONOCIMIENTOS TIC}

La incorporación de las TIC en época de Pandemia de la Covid-19, movilizó la práctica docente y el diseño curricular estuvo determinado por diferentes aspectos que condicionan de manera determinante su correcta implementación; un conjunto de saberes, conocimientos, capacidades, valores, creencias y actitudes que contribuya al uso adecuado de software y hardware que permitan la búsqueda, organización y acceso a la información al servicio de la construcción de conocimiento y valoración de actitudes en los Docentes (Cardona, Rivas \& Ramírez, 2020)

Figura 2. Modelo TPACK

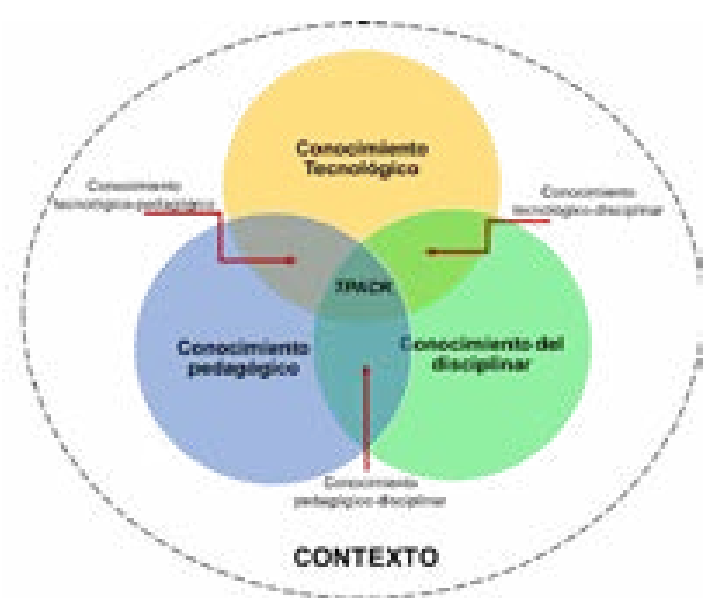

Fuente: (Koehler et al., 2009, 2015). 
La cultura digital y la información disponible en este medio, nos lleva a evidenciar la necesidad imperante de una formación docente que desarrolle en él, una competencia digital que propicie escenarios virtuales, el uso de herramientas tecnológicas, programas y nuevas aplicaciones, de allí la importancia de este saber y su fortalecimiento como competencia del docente de cara a la realidad actual, son las principales incidencias de un aprendizaje investido que trabajo sobre un modelo que entre a permear la TIC como mediación en el proceso de enseñanza-aprendizaje.

Desde una perspectiva más global, se desarrolló el modelo TPACK que buscaba integrar el conocimiento tecnológico, el pedagógico y el correspondiente a los contenidos específicos del ámbito de actuación (Figura 2). Lo significativo que propone el modelo es que el docente debe estar capacitado para incluir las TIC en el contexto formativo, teniendo en cuenta la interacción del conocimiento tecnológico, pedagógico y disciplinar (Samperio \& Barragán, 2018).

Las interacciones entre los conocimientos establecen diálogos entre los conceptos propios de las áreas y aborda conceptos sobre pedagogía y su relación con las TIC con el propósito de facilitar la creación de conocimiento de la disciplina en la educación superior (Cardona et al., 2020) La investigación en este modelo TPACK de Koehler et al. (2017) pone de manifiesto diferentes aspectos entre los que cabe subrayar:

$\checkmark \quad$ Actitudes diferenciadas de los docentes en formación -motivados y no motivados.

$\checkmark \quad$ Importancia de las oportunidades que ofrece la institución formativa para que los alumnos y alumnas desarrollen su modelo TPACK. $\checkmark \quad$ Necesidad de incluir la tecnología como un proceso sistémico y sistemático en la capacitación docente.

$\checkmark \quad$ Importancia del trabajo en equipo, en particular para aquellos docentes en formación con una percepción baja de competencia en TIC.

$\checkmark \quad$ Validez del trabajo en relación con el diseño de materiales curriculares soportados en tecnologías para los y las docentes en formación.

El modelo TPACK ha evolucionado hacia el modelo PEAT (Figura 3) que añade una dimensión ética y otra actitudinal para el uso de tecnologías digitales en contextos profesionales.

El modelo consiste en cuatro dimensiones de igual importancia que permite integrar conocimientos, cuestiones éticas, competencias y habilidades digitales y la adaptabilidad de nuevas tecnologías (Cardona et al., 2020).

Figura 3. Modelo PEAT

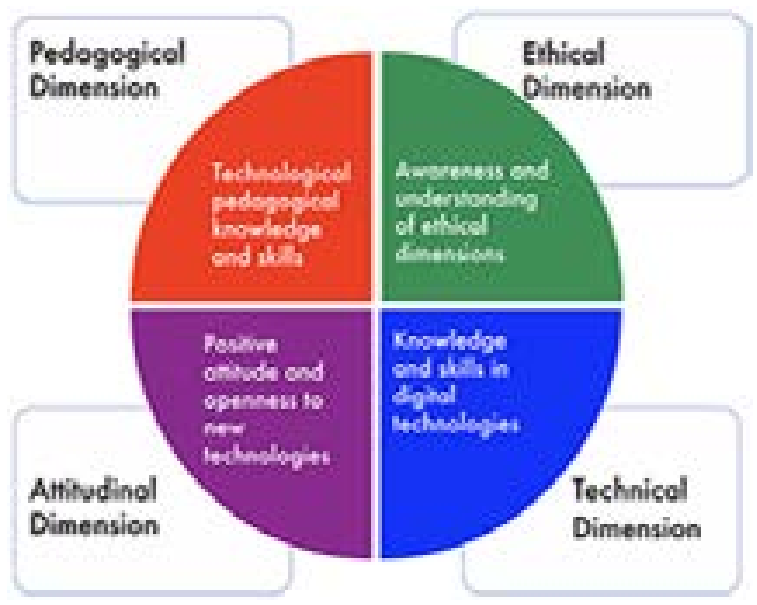

Fuente: (DICTE, 2019). 


\section{EL MODELO CONOCIMIENTO DEL CONTENIDO PEDAGÓGICO AMBIENTAL- EPACK}

\section{El Environmental Pedagogical Content} Knowledge-EPACK ${ }^{4}$ (Zhou, 2015) que se puede traducir como: Conocimiento del Contenido Pedagógico Ambiental presenta una interacción entre tres dominios de conocimiento: Conocimiento Ambiental (CA), Conocimiento de Contenido (CC) y Conocimiento Pedagógico (CP). Este modelo brinda oportunidades de aprendizaje mediante la incorporación de la dimensión ambiental, e implica una enseñanza que promueva y enriquezca la integración significativa de cuestiones ambientales en el plan de estudios de la asignatura (Zhou, 2015). Estos tres dominios interactúan dando como resultado diferentes formas de conocimiento combinado para, finalmente llegar al Conocimiento del Contenido Pedagógico como lo muestra la Figura 4.

Figura 4. Modelo EPACK

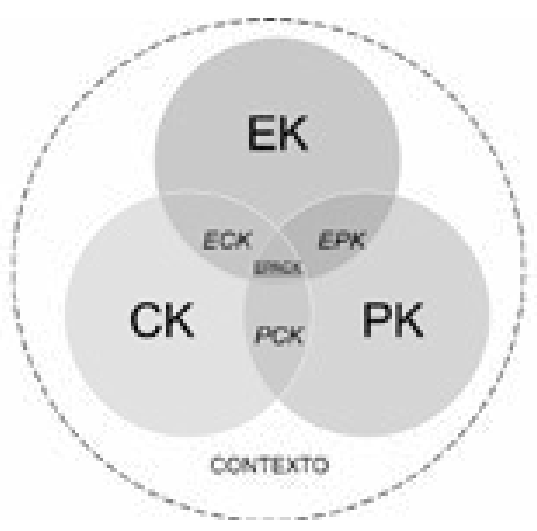

Fuente: (Zhou, 2015)

El modelo EPACK maneja las interacciones entre los tres dominios generan diferentes formas de conocimiento combinado: Conocimiento ambiental del contenido (ECK):

$4 \quad$ Modelo EPACK es el Conocimiento del contenido pedagógico ambiental, donde EK: Conocimiento Ambiental, CK: Conocimiento de Contenido, PK: Conocimiento Pedagógico, ECK: Conocimiento del contenido ambiental, EPK: Conocimiento pedagógico Ambiental, PCK: Conocimiento Pedagógico del Contenido
Se refiere al conocimiento de los candidatos a docentes acerca de la conexión entre los problemas ambientales y los temas requeridos por el plan de estudios.

Para integrar la educación ambiental en la enseñanza de su materia, el docente debe abordar temas ambientales que generen espacios apropiados en el campo de enseñanza.

Donnelly \& Berry (2019) citado por Zhou, (2015), afirman que el estado del ECK (Environmental content knowledge- siglas en ingles) de un docente determina la forma en que asesora los estudiantes y los orientan sobre cómo los miembros de su disciplina interactúan con el mundo y la cultura que los rodea.

Conocimiento pedagógico ambiental (EPK): Incluye el papel que cumple la pedagogía dentro de las temáticas ambientales, y como junto con el aprendizaje pueden cambiar e incorporar diferentes cuestiones ambientales en los planes de estudio. Debido a que el abordaje de problemas ambientales puede llegar a contener términos de complejidad, incertidumbre e intangibilidad (Zhou, 2015).

$\mathrm{Y}$, por último, el Conocimiento pedagógico del contenido (PCK): Es el conocimiento del contenido transformado para la enseñanza. A través de este, el docente consigue reflexionar, razonar y retroalimentar su ejercicio pedagógico para hacerlo más efectivo.

Para la educación superior, el docente universitario debe construir, transformar y conjugar los contenidos disciplinares con sus experiencias personales y práctica profesional para facilitar la comprensión y el razonamiento de la materia en sus estudiantes que guiará al docente en cómo enseñar una disciplina, el cual es fruto de la experiencia en el aula (Cardona, Ramírez \& Rivas, 2020). 
La combinación de la formación en conocimientos especializados y la incorporación de temas ambientales dentro de una formación pedagógica hace parte del desarrollo de la competencia de los docentes para tener en cuenta los objetivos de una educación ambiental (Zhou, 2015).

La inclusión de las TIC en la educación superior a través de políticas institucionales de innovación, mejoras en la comunicación interinstitucional, logística e infraestructura conlleva a una transformación en la manera en que se ha desarrollado el proceso de aprendizaje y para promover la incorporación de la educación ambiental entendida como un eje transversal que impregna el quehacer universitario.

En relación con la implementación de las TIC en el campo de la educación, existen revelaciones importantes que indican la pertinencia de la integración conjunta entre docente, alumno, contenido y, cómo las relaciones entre estas formas de organización y su interacción, es

determinante al momento de explorar las bondades de las TIC y su implementación en el proceso de enseñanza aprendizaje para alcanzar su potencial transformador.

Así mismo, evidencia la diferencia entre el simple uso real o efectivo de las TIC, las diferentes formas de organización de actividades vinculadas entre docentes y alumnos $y$, un diseño tecno pedagógico para una correcta integración de las TIC en el quehacer docente y los procesos de enseñanza - aprendizaje.

\section{A MANERA DE REFLEXIÓN}

El aspecto teórico de los saberes-otros y la didáctica como un aspecto práctico, siendo el primero el relacionado con los conocimientos que elabora sobre procesos de enseñanza y aprendizaje y, el segundo, fundamentado en la aplicación de tales conocimientos, en su intervención efectiva en los procesos reales de enseñanza - aprendizaje, conduce a una reflexión crítica sobre las formas de abordar la Educación Superior del Siglo XXI.

La articulación con las TIC implica reconocer que pueden ser herramientas que contribuyan a la comprensión de la complejidad, que hacen parte del instrumental educativo necesario para transmitir eficazmente y asumir la herencia cultural de la época, así lo menciona el acceso a la TIC por parte de los profesionales de la educación tiene que operar como garantía de democratización. Se trata de restituir al sujeto de la educación un lugar protagónico en sus procesos de adquisición y consiguiente apropiación, transformación y uso de los saberes y conocimientos en juego en cada época.

Sumado a lo anterior, es importante resaltar que más allá de las TIC, es el desarrollo de las capacidades del hombre y cómo las orienta al servicio de un mundo mejor; restituir un lugar protagónico al sujeto educativo, es decir priorizarlo, considerarlo más importante que las TIC, dotarlo no sólo de la capacidad de uso, sino de la comprensión que le permita dominarlas para dejar de ser dominado.

Y por último es necesario que la Educación Superior esté permeada por una dimensión ambiental que permita comprender las realidades del desarrollo a escala humana $y$ este en armonía con las contribuciones de la naturaleza para la gente, propendiendo por el Buen Vivir/Vivir Bien, será el camino en la 
integración de una reforma universitaria con sentido crítico y para la realidad del mundo cambiante.

Son los modelos didácticos base para un proceso de formación integral de los Docentes Universitarios, con posibilidades de incluir esos saberes-otros, de tal manera que la consigna con la que comienza la comunicación puede ser validada en su completitud.

\section{REFERENCIAS BIBLIOGRÁFICAS}

Cardona, CM.; Ramírez, M.; Rivas., E. (2020). Educación Superior en un mundo virtual, forzado por la Pandemia de la Covid-19. Revista Espacios. Volumen 41. No. 35.

Garritz, A y Trinidad-Velasco, R. (2004). conocimiento pedagógico del contenido. Education Química. Volumen 15. No. 2. Abril. pp. 98-102.

Londoño-Orozco, Guillermo (2014). Docencia Universitaria: sentidos, didáctica, sujetos y sabereas. Volumen 6. (Editor). Universidad de La Salle. Vicerrectoría Académica. Bogotá D.C - Colombia. 478p.

Koehler, M. J., \& Mishra, P. (2009). What is technological pedagogical content knowledge? Contemporary issues in technology and teacher education, 9(1), 60-70.

Mora, W. y Parga, D. (2014). Aportes del CDC desde el pensamiento complejo. En: A., Garritz; S. Daza, y M. Lorenzo (Ed). Conocimiento didáctico del contenido: una perspectiva Iberoamericana (pp. 100-143). Saarbrücken, Alemania: Editorial Académica Española.
Morín, E. (1999). Los siete saberes necesarios para la educación del futuro. Organización de las Naciones Unidas para la Educación, la Ciencia y la Cultura. Paris - Francia

Ramírez, M; Rivas, E; Cardona, CM. (2019). El Estudio de caso como estrategia metodológica. Revista Espacios. Vol. 40 (№23), Pág. 30.

Shulman, L. S. (1987). Knowledge and teaching: foundations of the reform. Harvard Educational Review, 57(1), 1-22.

Zhou, G. (2015). Environmental Pedagogical Content Knowledge: A Conceptual Framework for Teacher Knowledge and Development. In: S. K. Stratton., R. Hagevik., A. Feldman, \& M. Bloom (Eds.) (2015). Educating Science Teachers for Sustainability. Chapter 11. (pp. 185 204). New York, London: Springer.

\section{REFERENCIAS SABERES-OTROS}

Corbetta, S., Sessano, P., \& Krasmanski, M. (2012). Educación ambiental, Formación de Docentes y TIC: El desafío complejo de una tripe articulación, hacia la definición de un espacio transversal. Escuela de Ciencias de la Educación.

Eschenhagen, M. L., \& López, F. (2016). Posibilidades para la ambientalización de la educación superior en América Latina: propuestas teóricas $y$ experiencias. Medellín, Colombia: Universidad pontificia Bolivariana. 
García, M. Á., Reyes, C. F., Merino, R. C., \& otros, y. (2014). La formación del Profesorado de Ciencias en Contextos de Diversidad: Una mirada desde la mediación con las TIC y la Construcción de Diseños Didácticos. Bogotá D.C, Cundinamarca, Colombia: UD-Editoriales. Serie Grupos.

Gudynas, E. (2014). Derechos de la Naturaleza y políticas Ambientales. (C. P.-S. Ambientales, Ed.)Bogotá D.C, Colombia: Jardín Botánico José Celestino Mutis Centro de Investigaciones y desarrollo Científico.

Imbernón-Muñoz, F. (2006). Actualidad y nuevos retos de la Formación Permanente. Revista Electrónica de Investigación Educativa, 8(2), 2-11.

Martínez, P. (2006). El método de Estudio de caso. Estrategia metodológica de la investigación científica (Vol. 20). Pensamiento y Gestión.

Mora, W. (2012). Ambientalización curricular: Un estudio cualitativo de las ideas del profesorado. Revista Currículo y formación del profesorado, 78-103.

Terrón-Amagón, E. (2013). Hacia una Educación Ambiental Crítica que articule la Interculturalidad: Modelo Pedagógico y Didáctico (Vol. 1). México: Horizonte Educativo

Yin, R. (2003). Case Study Research: Design and Methods (Third Edition ed.). (S. Publicaciones, Ed.). 